\title{
Probing Surface-Bound Atoms with Quantum Nanophotonics
}

\author{
Daniel Hümmer® ${ }^{1,2}$ Oriol Romero-Isart $\oplus^{1,2}$ Arno Rauschenbeutel ${ }^{3}{ }^{3}$ and Philipp Schneeweiss $\oplus^{3,4, *}$ \\ ${ }^{1}$ Institute for Quantum Optics and Quantum Information of the Austrian Academy of Sciences, 6020 Innsbruck, Austria \\ ${ }^{2}$ Institute for Theoretical Physics, University of Innsbruck, 6020 Innsbruck, Austria \\ ${ }^{3}$ Department of Physics, Humboldt-Universität zu Berlin, 10099 Berlin, Germany \\ ${ }^{4}$ Atominstitut, TU Wien, 1020 Vienna, Austria
}

(Received 13 July 2020; accepted 29 March 2021; published 22 April 2021)

\begin{abstract}
Quantum control of atoms at ultrashort distances from surfaces would open a new paradigm in quantum optics and offer a novel tool for the investigation of near-surface physics. Here, we investigate the motional states of atoms that are bound weakly to the surface of a hot optical nanofiber. We theoretically demonstrate that with optimized mechanical properties of the nanofiber these states are quantized despite phononinduced decoherence. We further show that it is possible to influence their properties with additional nanofiber-guided light fields and suggest heterodyne fluorescence spectroscopy to probe the spectrum of the quantized atomic motion. Extending the optical control of atoms to smaller atom-surface separations could create opportunities for quantum communication and instigate the convergence of surface physics, quantum optics, and the physics of cold atoms.
\end{abstract}

DOI: 10.1103/PhysRevLett.126.163601

Obtaining optical control over individual atoms close to surfaces would enable significant advances in fundamental research. For instance, trapping atoms closer to a waveguide increases their coupling to the guided light fields. The increased emission into the waveguide aids the exploration of novel effects in quantum optics [1] and benefits powerful light-matter interfaces useful for quantum communication [2]. Moreover, the measurement precision of effects in surface and near-surface physics such as dispersion forces could profit from isotopically clean atomic probes with well-defined initial conditions and long interrogation times [3-8]. A detailed understanding of atom-surface interactions is paramount, for example, in the search for nonNewtonian forces [9] or surface-induced friction [10]. Precise control over the motional and electronic degrees of freedom of atoms near surfaces would, therefore, provide advantages for quantum optics and surface physics and could ultimately enable the transfer of techniques between these two disparate fields. At present, cold atoms can be optically trapped at distances of a few hundred nanometers from surfaces [11-20]. At shorter distances, attractive dispersion forces dominate over conventional traps and can lead to adsorption [21]. Conversely, the omnipresence of dispersion forces has stimulated ideas to exploit them for trapping atoms in the first place [22-24]. In previous works

Published by the American Physical Society under the terms of the Creative Commons Attribution 4.0 International license. Further distribution of this work must maintain attribution to the author(s) and the published article's title, journal citation, and DOI. on the optical control of adsorbed atoms [25-30], it remained unclear whether the motional states are quantized despite decoherence [31-33], and how to optimally probe and manipulate this system.

Here, we propose an experiment to optically detect the quantized motion of atoms bound directly to the surface of a waveguide. We consider two cases: adsorbed atoms and surface-bound atoms in a hybrid potential created by adding an attractive optical force. We focus on weakly bound motional states with binding energies corresponding to a few megahertz since these states can efficiently be probed with light. We account for the finite linewidth of transitions between motional states, which is caused by thermal vibrations (phonons) of the waveguide. We identify a parameter regime in which the atomic motion normal to the surface is quantized despite the interaction with phonons. Interestingly, the linewidths are limited by phonon-induced dephasing rather than state depopulation. We further show that the spectrum of the quantized atomic motion can be resolved using heterodyne fluorescence spectroscopy.

We consider cesium atoms bound to a silica nanofiber [34-36] for the sake of concreteness. The existence of adsorbed states of cesium on silica is undisputed [37-39]. However, the quantization of the adatoms' motion normal to the surface can only be observed if transitions between different motional states have linewidths smaller than the splitting between the transition frequencies in the absence of vibrations. The interaction with phonons is the dominant mechanism causing depopulation both for adsorbed $[31,32]$ and optically trapped atoms [40], and leads to dephasing as well. We assume that the nanofiber forms a phonon cavity of length $L$. Such a cavity provides control over the 
nanofiber phonon modes and could, for instance, be realized by optimizing the nanofiber tapers [41]. To calculate the total linewidth of transitions between the motional states of an individual atom, we describe the coupled dynamics of the atomic motion and the nanofiber phonons using the Hamiltonian

$$
\hat{H}=\hat{H}_{\text {ext }}+\hat{H}_{\text {phn }}+\hat{H}_{\text {ext-phn }} .
$$

The atom Hamiltonian $\hat{H}_{\text {ext }}=\hat{\boldsymbol{p}}^{2} /(2 M)+V(\hat{r})$ describes the motion of the atom of mass $M$ in the adiabatic potential $V(r)$. The operator $\hat{r}$ represents the distance of the atom from the axis of nanofiber and $\hat{\boldsymbol{p}}$ the momentum of the atom. The term $\hat{\boldsymbol{H}}_{\mathrm{phn}}$ describes the dynamics of the nanofiber phonons, and the term $\hat{\boldsymbol{H}}_{\text {ext-phn }}$ accounts for the atom-phonon coupling. It is sufficient to treat each atom individually since the far-detuned probe laser subsequently used for the spectroscopy does not induce long-ranged atom-atom interactions mediated by the exchange of resonant waveguide photons [42-44].

The potential $V(r)$ arises from both optical dipole forces $[45,46]$ and surface effects $[33,47]$. We approximate the total potential as $V(r)=V_{\text {opt }}(r)+V_{\text {ad }}(r)$. Nonadditive corrections are only relevant for sufficiently strong light fields [48]. The potential $V_{\text {opt }}(r)$ can be calculated $[46,49,50]$. In contrast to nanofiber-based two-color traps $[15,16]$, we consider a cylindrically symmetric potential without a repulsive optical force to prevent the atom from accessing the nanofiber surface. The adsorption potential $V_{\text {ad }}(r)$ is determined by the choice of atom species and nanofiber material. It is predominantly due to the CasimirPolder interaction and the exchange interaction [21,51,52]. The attractive Casimir-Polder force (dispersion force) dominates over optical forces at atom-surface separations below a few tens of nanometers [46,47]. The exchange interaction becomes relevant when electrons orbiting the atom begin to overlap with electrons in the nanofiber surface $[21,51,53]$. It causes a strong repulsion of the atom immediately at the nanofiber surface. We model the adsorption potential as

$$
V_{\mathrm{ad}}(r)=-C(r-R)^{-3}+D(r-R)^{-12} .
$$

Here, $r$ is the radial distance of the atom from the nanofiber axis and $R$ is the radius of the nanofiber; see the inset in Fig. 1(a). The first term in Eq. (2) is the dispersion force between an atom and a half-space. This simplified model neglects effects such as retardation and the nanofiber's cylindrical geometry, which do not qualitatively alter the results presented in the following [54]. The constant $C>0$ can be calculated $[56,59,60]$ and determined experimentally. For a cesium atom and a silica surface $C / h=1.18 \mathrm{THz} \mathrm{nm}^{3}$ [61], where $h$ is Planck's constant. The second term in Eq. (2) is a standard heuristic model for the exchange energy [53]. The constant $D>0$ can be

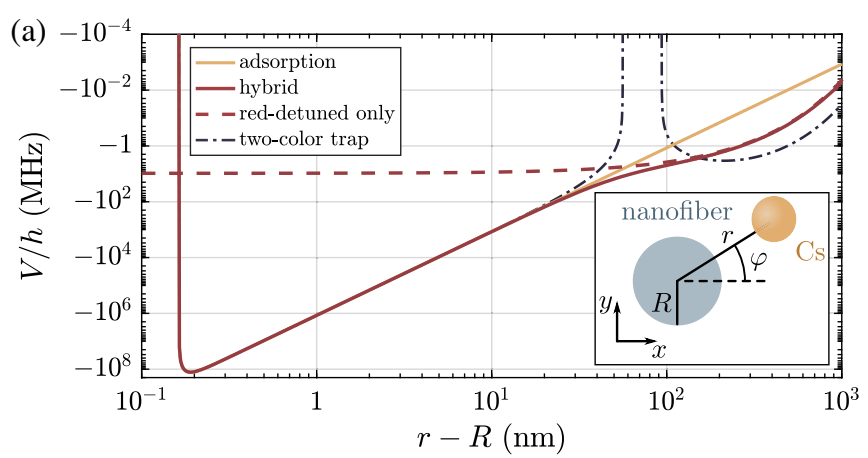

(b)

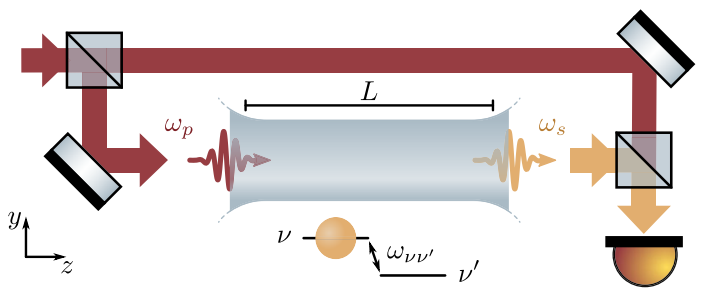

FIG. 1. Panel (a) shows the adiabatic potential as a function of the atom-surface separation. The yellow line represents the adsorption potential $V_{\text {ad }}$, the red line a hybrid light- and surface-induced potential, and the red dashed line the contribution of the optical potential $V_{\text {opt }}$. The dash-dotted line corresponds to a typical two-color optical trap for comparison. The inset illustrates some key experimental parameters. Panel (b) outlines the proposed setup for heterodyne fluorescence spectroscopy of the motional states.

inferred from the minimum $V_{\min }$ of the adsorption potential $V_{\text {ad }}(r)$. We use $V_{\min } / h=-128 \mathrm{THz}[37,38]$, which yields $D / h=96.5 \mathrm{kHz} \mathrm{nm}^{12}$. Importantly, the bound state energies and spectral peaks presented in Figs. 2 and 3 quantitatively depend on the parameters $C, V_{\min }$, and the exponent $p=-12$ used in Eq. (2) and hence provide information about the atom-surface interaction. At the same time, our findings are qualitatively independent of these details and still hold when using alternative models like an exponential barrier [53] for the short-range repulsive interaction [62].

In Fig. 1(a), we plot the potential $V(r)$. The hybrid lightand surface-induced potential is realized by launching into the nanofiber a circularly polarized, guided, running-wave light field with a free-space wavelength of $1064 \mathrm{~nm}$ (red detuned relative to the cesium $D_{2}$ line) and a power $P_{r}=1 \mathrm{~mW}$. We also show the potential of a typical nanofiber-based two-color optical dipole trap for comparison; see the Supplemental Material for details [63]. We assume a relative permittivity of $\epsilon=2.1$ [79] and a nanofiber radius of $R=305 \mathrm{~nm}$ [80].

The radial motional states have frequencies $\omega_{\nu}$ and wave functions $\psi_{\nu}(r) \equiv \sqrt{r}\langle r \mid \nu\rangle$ that are obtained by solving the time-independent Schrödinger equation:

$$
\left[-\frac{\hbar^{2}}{2 M} \partial_{r}^{2}+V(r)\right] \psi_{\nu}(r)=\hbar \omega_{\nu} \psi_{\nu}(r)
$$



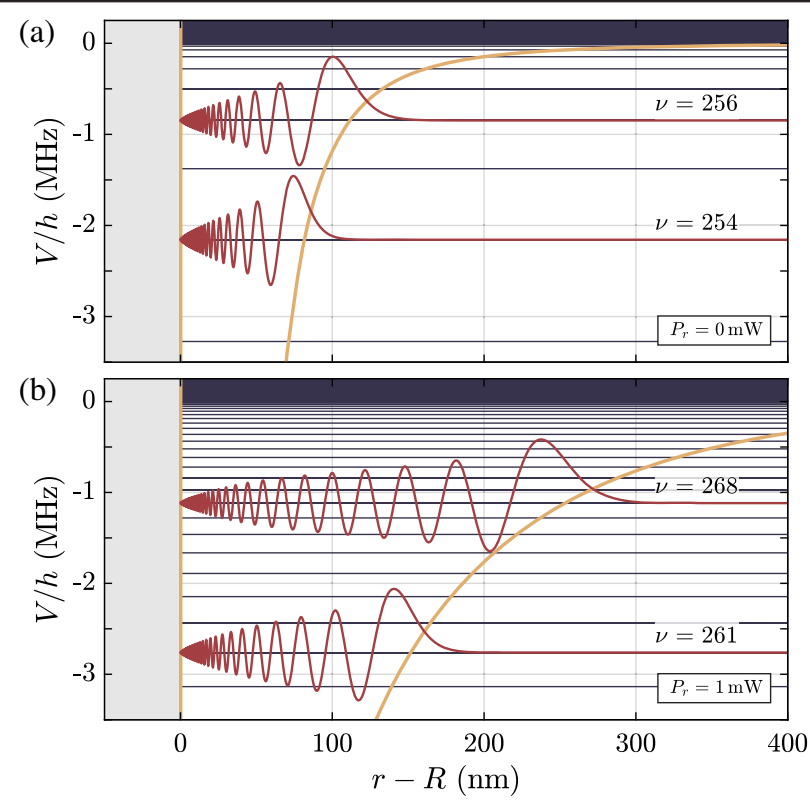

FIG. 2. Radial motional states of a cesium atom bound to a silica nanofiber. Panel (a) shows adsorbed states, panel (b) hybrid surface-bound states. We plot the corresponding potential $V$ (yellow) generated at power $P_{r}$ of the fiber-guided light beam, the spectrum $\omega_{\nu} / 2 \pi$ of motional states (dark blue), and two examples of the atom wave function (red) in arbitrary units. The gray area at $r-R<0$ marks the position of the nanofiber.

Here, the index $\nu$ counts the motional quanta in radial direction. The motion in azimuthal and axial direction can be neglected [63], so $\hat{H}_{\mathrm{ext}}=\hbar \sum_{\nu} \omega_{\nu}|\nu\rangle\langle\nu|$. We solve
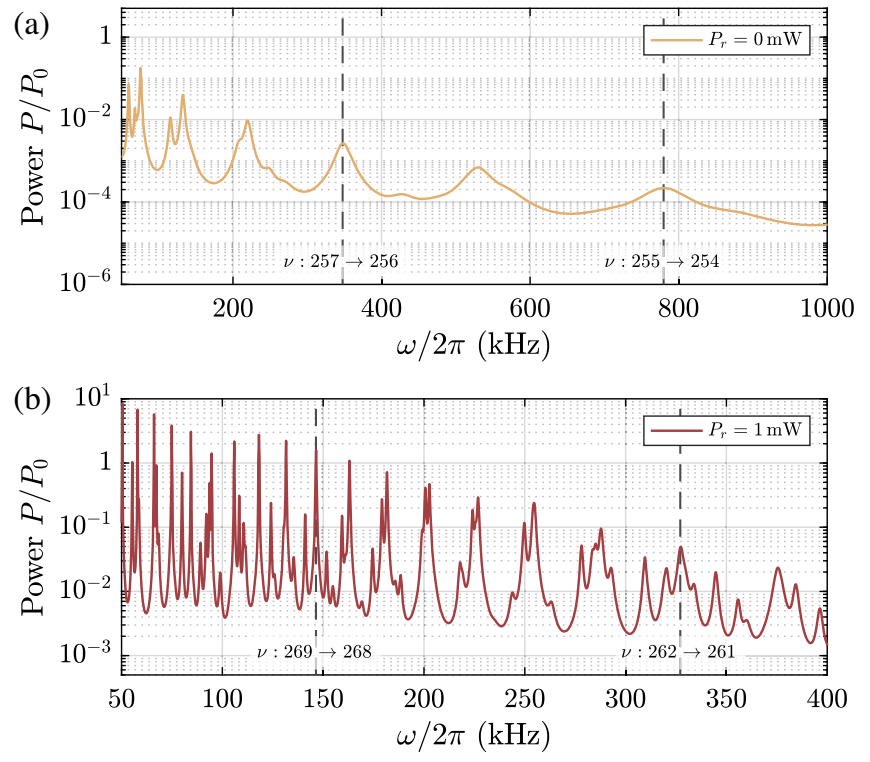

FIG. 3. Spectrum of light inelastically scattered by nanofiberbound atoms. We plot the power of the scattered light as a function of the frequency difference $\omega=\omega_{s}-\omega_{p}$ of the probe photon and the scattered photon. The scale $P_{0}$ is explained in the text. Panels (a) and (b) show sidebands due to transitions between the states in Figs. 2(a) and 2(b), respectively.
Eq. (3) numerically [81]. In Fig. 2, we plot the spectrum $\omega_{\nu}$ and some example wave functions $\psi_{\nu}(r)$ using $M=2.21 \times 10^{-25} \mathrm{~kg}$ [83]. Figure 2(a) shows weakly bound states with binding energies up to a few megahertz. Figure 2(b) shows surface-bound states in the hybrid lightand surface-induced potential. While the expected centerof-mass position of an atom in these states is on the order of $100 \mathrm{~nm}$, there is no potential barrier to keep the atom from accessing the surface.

The phonon Hamiltonian is $\hat{H}_{\mathrm{phn}}=\hbar \sum_{\mu} \omega_{\mu} \hat{b}_{\mu}^{\dagger} \hat{b}_{\mu}$, where $\mu$ is an index labeling the phonon modes and $b_{\mu}$ are the corresponding bosonic ladder operators. The phonon modes of a nanofiber can be calculated analytically $[63,84]$. The depopulation of the motional states in nanofiber-based two-color traps is dominated by their interaction with flexural phonon modes [40]. The coupling primarily arises because the moving nanofiber surface displaces the adiabatic potential [40]. The atom experiences the shifted potential $V\left[\hat{r}-\hat{u}^{r}(R, \hat{\varphi}, \hat{z})\right][32,33]$, where $\hat{u}^{r}$ is the radial displacement of the nanofiber surface and $\hat{\boldsymbol{r}}=(\hat{r}, \hat{\varphi}, \hat{z})$ is the position operator of the atom in cylindrical coordinates. To describe depopulation and dephasing, we expand the potential to second order in the phonon field. The zero-order term appears in $\hat{H}_{\text {ext }}$, while higher orders form the interaction Hamiltonian $\hat{H}_{\text {ext-phn }} \simeq \hat{H}_{\text {ext-phn }}^{(1)}+\hat{H}_{\text {ext-phn }}^{(2)}$. At first order,

$$
\hat{H}_{\text {ext-phn }}^{(1)}=\hbar \sum_{\mu \nu^{\prime} \nu}\left(g_{\mu \nu^{\prime} \nu} \hat{b}_{\mu}\left|\nu^{\prime}\right\rangle\langle\nu|+\text { H.c. }\right) \text {. }
$$

At second order, we only retain terms describing resonant elastic two-photon scattering, which yield the principal second-order contribution to the broadening of motional transitions [63]:

$$
\hat{H}_{\mathrm{ext}-\mathrm{phn}}^{(2)}=\hbar \sum_{\mu \nu} G_{\mu \nu} \hat{b}_{\mu}^{\dagger} \hat{b}_{\mu}|\nu\rangle\langle\nu| .
$$

The coupling rates are

$$
g_{\mu \nu^{\prime} \nu}=\frac{i}{\sqrt{2 \pi}} \frac{\mathcal{A}^{(1)}{ }_{\nu^{\prime} \nu}}{\sqrt{\hbar \rho \omega_{\mu} L},}, \quad G_{\mu \nu}=\frac{1}{2 \pi} \frac{\mathcal{A}^{(2)}{ }_{\nu \nu}}{\rho \omega_{\mu} L R^{2}},
$$

where $\rho$ is the density of the nanofiber $\left(\rho=2.20 \mathrm{~g} / \mathrm{cm}^{3}\right.$ for fused silica [79]), and we define the phonon-induced overlap between different states:

$$
\mathcal{A}_{\nu^{\prime} \nu}^{(i)} \equiv \int_{0}^{\infty} \psi_{\nu^{\prime}}^{*}(r) \psi_{\nu}(r) \partial_{r}^{i} V(r) d r
$$

The wave functions $\psi_{\nu}(r)$ are normalized according to the orthonormality condition $\int_{0}^{\infty} \psi_{\nu}^{*}(r) \psi_{\nu^{\prime}}(r) d r=\delta_{\nu \nu^{\prime}}$, where $\delta$ is the Kronecker symbol. The coupling rates are small compared to the transition frequencies $\omega_{\nu^{\prime} \nu} \equiv \omega_{\nu^{\prime}}-\omega_{\nu}$; that is, $\left|\omega_{\nu^{\prime} \nu}\right| \gg\left|g_{\mu \nu^{\prime} \nu}\right|,\left|G_{\mu \nu}\right|$. Assuming further that the phonon 
modes have large decay rates $\kappa_{\mu} \gg\left|g_{\mu \nu^{\prime} \nu}\right|,\left|G_{\mu \nu}\right|$ compared to the coupling rates, the phonon modes can be adiabatically eliminated to obtain an effective description of the atom motion in the presence of the thermal phonon bath [63].

One can then show that if a transition $\nu^{\prime} \leftrightarrow \nu$ between different motional states is externally driven, its resonance has a finite phonon-induced linewidth (full width at half maximum) of

$$
\Gamma_{\nu^{\prime} \nu}=\Gamma_{\nu^{\prime} \nu}^{(1)}+\Gamma_{\nu^{\prime} \nu}^{(2)}
$$

see Ref. [63]. Here, $\Gamma_{\nu^{\prime} \nu}^{(1)}=\Gamma_{\nu^{\prime}}^{d}+\Gamma_{\nu}^{d}$ is the broadening due to depopulation of the two motional states caused by phonon absorption and emission through $\hat{H}_{\text {ext-phn }}^{(1)}$. The depopulation rate $\Gamma_{\nu}^{d} \simeq \Gamma_{\nu}^{-}+\Gamma_{\nu}^{+}$of each state is dominated by transitions to the nearest neighboring states. It is beneficial to work with a short phonon cavity to minimize $\Gamma_{\nu^{\prime} \nu}$. For our case study, we choose a cavity sufficiently small such that the frequency $\omega_{1}=\pi^{2} R \sqrt{E / \rho} /\left(2 L^{2}\right)$ of the fundamental cavity mode $\mu_{1}$ is larger than the transition frequencies $\left|\omega_{(\nu \pm 1) \nu}\right|$ of interest. Here, $E$ is the Young's modulus of the nanofiber ( $E=72.6 \mathrm{GPa}$ for fused silica [79]). In this limit, $\Gamma_{\nu}^{ \pm}$is determined by the nonresonant coupling to the fundamental mode. As a result,

$$
\Gamma_{\nu}^{ \pm} \simeq 4 \bar{n} \frac{\left|g_{\mu_{1}(\nu \pm 1) \nu}\right|^{2}}{\omega_{1}} \frac{1}{Q}
$$

where $\bar{n}$ is the thermal population and $Q=\omega_{1} / \kappa_{1}$ the quality factor. In deriving Eq. (9), we assume $\bar{n} \simeq k_{B} T / \hbar \omega_{1} \gg 1$, where $T$ is the temperature of the nanofiber and $k_{B}$ is the Boltzmann constant. The second contribution in Eq. (8),

$$
\Gamma_{\nu^{\prime} \nu}^{(2)} \simeq 16 \bar{n}^{2} \frac{\left|G_{\mu_{1} \nu^{\prime} \nu}\right|^{2}}{\omega_{1}} Q,
$$

is primarily caused by dephasing between the motional states due to the resonant coupling to the fundamental mode through $\hat{H}_{\text {ext-phn }}^{(2)}$. Here, $G_{\mu_{1} \nu^{\prime} \nu} \equiv\left(G_{\mu_{1} \nu^{\prime}}-G_{\mu_{1} \nu}\right) / 2$. We assume a cavity of length $L=5 \mu \mathrm{m}$ and quality factor $Q=100$. In this case, the linewidth is limited by dephasing; that is, $\Gamma_{\nu^{\prime} \nu}^{(2)} \gg \Gamma_{\nu^{\prime} \nu}^{(1)}$. Remarkably, $\Gamma_{\nu^{\prime} \nu}$ can be small enough such that transitions between the motional states shown in Fig. 2 can be resolved as we now argue.

We propose to measure the spectrum of the quantized nanofiber-bound states using heterodyne fluorescence spectroscopy, see Fig. 1(b), which allows the observation of the quantized motion of atoms in optical potentials [85]. To this end, a cloud of laser-cooled atoms is prepared around the nanofiber. The nanofiber-bound states are in a thermal equilibrium [31,32]. Laser light with a frequency $\omega_{p}$ far detuned from resonance with the atom is split into a probe beam and a local oscillator; see Fig. 1(b). The probe beam is coupled into the nanofiber with circular polarization. A guided probe photon can be scattered inelastically by a bound atom through the evanescent electric field, changing its frequency to $\omega_{s}$ and causing the atom to change its motional state from $\nu$ to $\nu^{\prime}$. This process creates sidebands in the spectrum of the probe beam. After the transmission through the nanofiber, the probe beam is recombined with the local oscillator. The beat signal is detected with a photodetector. The frequency of the local oscillator is shifted by an offset $\Delta \omega$ to separate the Stokes and anti-Stokes sidebands, and its polarization is matched to that of the probe beam. This setup is only sensitive to the radial motion of bound atoms [63]. The power $P$ of the scattered light as a function of the difference $\omega \equiv \omega_{s}-\omega_{p}$ can be inferred from the spectrum of the photocurrent.

The spectroscopy can be modeled by the Hamiltonian

$$
\hat{H}^{\prime}=\hat{H}+\hat{H}_{\text {int }}+\hat{H}_{\text {pht }}+\hat{H}_{\text {int-pht }},
$$

where $\hat{H}_{\mathrm{pht}}=\hbar \sum_{\eta} \omega_{\eta} \hat{a}_{\eta}^{\dagger} \hat{a}_{\eta}$ describes the nanofiber-guided photon modes $\eta$ and $\hat{H}_{\text {int-pht }}=-\hat{\boldsymbol{d}} \cdot \hat{\boldsymbol{E}}(\hat{\boldsymbol{r}})$ is the dipole coupling [63]. Here, $\hat{\boldsymbol{E}}$ is the electric field and $\hat{\boldsymbol{d}}$ is the dipole moment of a single atom. One can show that the power of scattered light as a function of the frequency difference $\omega$ is approximately [63]

$$
P(\omega) \propto \sum_{\nu, \nu^{\prime} \neq \nu} \frac{\Gamma_{\nu^{\prime} \nu} / 2}{\left(\omega_{\nu^{\prime} \nu}-\omega\right)^{2}+\left(\Gamma_{\nu^{\prime} \nu} / 2\right)^{2}} n(\nu)\left|\mathcal{F}_{\nu^{\prime} \nu}\right|^{2} .
$$

Since the potential $V(r)$ is not harmonic, this spectrum contains a separate sideband for each transition $\nu \leftrightarrow \nu^{\prime}$. The amplitude of each sideband is proportional to the FranckCondon factor,

$$
\mathcal{F}_{\nu^{\prime} \nu} \equiv \frac{E_{\eta_{s}} E_{\eta_{p}}}{(2 \pi)^{2}} \int_{0}^{\infty} \psi_{\nu^{\prime}}^{*}(r) \mathcal{E}_{\eta_{s}}^{*}(r) \cdot \mathcal{E}_{\eta_{p}}(r) \psi_{\nu}(r) d r,
$$

where we define $E_{\eta} \equiv \sqrt{\hbar \epsilon_{0} \omega_{\eta} / 2}$. Here, $\epsilon_{0}$ is the vacuum permittivity, the index $\eta_{p}\left(\eta_{s}\right)$ comprises the quantum numbers of the nanofiber-guided probe (scattered) photon, and $\mathcal{E}_{\eta}(r)$ is the radial partial wave of the corresponding electric mode field of the fundamental $\mathrm{HE}_{11}$ mode of a nanofiber [86-88].

In Fig. 3(a), we plot the anti-Stokes sidebands corresponding to downward transitions between the adsorbed states shown in Fig. 2(a), assuming a nanofiber temperature of $T=300 \mathrm{~K}$. The spectrum in Fig. 3(b) corresponds to the hybrid surface-bound states shown in Fig. 2(b), assuming $T=420 \mathrm{~K}$ based on the power $P_{r}$ [89]. In both cases, transitions between neighboring levels are resolved. Examples of such transitions are indicated by the dashed lines. Transitions between levels that are further separated in $\nu$ appear as smaller, interstitial peaks. In plotting Fig. 3, 
we choose a wavelength of $1000 \mathrm{~nm}$ for the probe laser and approximate the occupation of all relevant states as equal since the frequency interval they cover is much smaller than $k_{B} T$. The signal decreases for larger $\omega$ since the involved states have a smaller spatial extent, resulting in lower Franck-Condon factors. For this reason, we focus on states with binding energies of a few megahertz. The additional red-detuned light field increases the scattering probability in Fig. 2(b) by widening the wave functions: The resonances highlighted in Figs. 2(a) and 3(b) involve states with similar binding energies, but the signal is increased in the latter case, boosting resonances above $P / P_{0}=1$. Here, $P_{0}$ is the power of the sideband corresponding to transitions between the first excited state $\nu=1$ and the ground state $\nu=0$ in the regular nanofiber-based two-color trap shown in Fig. 1 [63], a signal that has already been observed experimentally [90].

In summary, we analyze the spectrum and phonon-induced linewidths of the motional states of a cesium atom bound directly to the surface of an optical nanofiber. We find that the phonon-induced linewidth of transitions between states with binding energies of a few megahertz can be smaller than the spacing of the transitions, allowing one to resolve quantized motional states. We further propose to probe these states using heterodyne fluorescence spectroscopy. An additional attractive light field enhances the expected signal compared to purely adsorbed atoms. When working at room temperature, it is necessary to optimize the nanofiber's mechanical properties to resolve the quantization of the motional states, which could explain why it has not previously been observed. The proposed technique can be adapted for other waveguide geometries, including chip-based implementations, and is expected to work for other combinations of atom species and waveguide materials.

We thank Jürgen Volz and Carlos Gonzalez-Ballestero for helpful discussions. Support by the Austrian Academy of Sciences (ÖAW, ESQ Discovery Grant QuantSurf), the Studienstiftung des Deutschen Volkes, and the Alexander von Humboldt Foundation in the framework of the Alexander von Humboldt Professorship endowed by the Federal Ministry of Education and Research is gratefully acknowledged.

*Corresponding author. philipp.schneeweiss@hu-berlin.de

[1] D. E. Chang, J. S. Douglas, A. González-Tudela, C.-L. Hung, and H. J. Kimble, Rev. Mod. Phys. 90, 031002 (2018).

[2] N. V. Corzo, J. Raskop, A. Chandra, A. S. Sheremet, B. Gouraud, and J. Laurat, Nature (London) 566, 359 (2019).

[3] Casimir Physics, edited by D. Dalvit, P. Milonni, D. Roberts, and F. da Rosa (Springer, Berlin, 2011).

[4] M. Gierling, P. Schneeweiss, G. Visanescu, P. Federsel, M. Häffner, D. P. Kern, T. E. Judd, A. Günther, and J. Fortágh, Nat. Nanotechnol. 6, 446 (2011).
[5] P. Schneeweiss, M. Gierling, G. Visanescu, D. P. Kern, T. E. Judd, A. Günther, and J. Fortágh, Nat. Nanotechnol. 7, 515 (2012).

[6] F. Yang, A. J. Kollár, S. F. Taylor, R. W. Turner, and B. L. Lev, Phys. Rev. Applied 7, 034026 (2017).

[7] M. Fichet, G. Dutier, A. Yarovitsky, P. Todorov, I. Hamdi, I. Maurin, S. Saltiel, D. Sarkisyan, M.-P. Gorza, D. Bloch, and M. Ducloy, Europhys. Lett. 77, 54001 (2007).

[8] T. Peyrot, N. Šibalić, Y. R. P. Sortais, A. Browaeys, A. Sargsyan, D. Sarkisyan, I. G. Hughes, and C. S. Adams, Phys. Rev. A 100, 022503 (2019).

[9] R. Onofrio, New J. Phys. 8, 237 (2006).

[10] F. Intravaia, V. E. Mkrtchian, S. Y. Buhmann, S. Scheel, D. A. R. Dalvit, and C. Henkel, J. Phys. Condens. Matter 27, 214020 (2015).

[11] M. Hammes, D. Rychtarik, H.-C. Nägerl, and R. Grimm, Phys. Rev. A 66, 051401(R) (2002).

[12] C. Stehle, H. Bender, C. Zimmermann, D. Kern, M. Fleischer, and S. Slama, Nat. Photonics 5, 494 (2011).

[13] J. D. Thompson, T. G. Tiecke, N. P. de Leon, J. Feist, A. V. Akimov, M. Gullans, A. S. Zibrov, V. Vuletić, and M. D. Lukin, Science 340, 1202 (2013).

[14] A. Goban, C.-L. Hung, J. D. Hood, S.-P. Yu, J. A. Muniz, O. Painter, and H. J. Kimble, Phys. Rev. Lett. 115, 063601 (2015).

[15] E. Vetsch, D. Reitz, G. Sagué, R. Schmidt, S. T. Dawkins, and A. Rauschenbeutel, Phys. Rev. Lett. 104, 203603 (2010).

[16] A. Goban, K. S. Choi, D. J. Alton, D. Ding, C. Lacroûte, M. Pototschnig, T. Thiele, N. P. Stern, and H. J. Kimble, Phys. Rev. Lett. 109, 033603 (2012).

[17] J.-B. Béguin, E. M. Bookjans, S. L. Christensen, H. L. Sørensen, J. H. Müller, E. S. Polzik, and J. Appel, Phys. Rev. Lett. 113, 263603 (2014).

[18] S. Kato and T. Aoki, Phys. Rev. Lett. 115, 093603 (2015).

[19] J. Lee, J. A. Grover, J. E. Hoffman, L. A. Orozco, and S. L. Rolston, J. Phys. B 48, 165004 (2015).

[20] N. V. Corzo, B. Gouraud, A. Chandra, A. Goban, A. S. Sheremet, D. V. Kupriyanov, and J. Laurat, Phys. Rev. Lett. 117, 133603 (2016).

[21] M.-C. Desjonqueres and D. Spanjaard, Concepts in Surface Physics (Springer, Berlin, 2012).

[22] C.-L. Hung, S. M. Meenehan, D. E. Chang, O. Painter, and H. J. Kimble, New J. Phys. 15, 083026 (2013).

[23] D. E. Chang, K. Sinha, J. M. Taylor, and H. J. Kimble, Nat. Commun. 5, 4343 (2014).

[24] A. González-Tudela, C.-L. Hung, D. E. Chang, J. I. Cirac, and H. J. Kimble, Nat. Photonics 9, 320 (2015).

[25] E. G. Lima, M. Chevrollier, O. Di Lorenzo, P. C. Segundo, and M. Oriá, Phys. Rev. A 62, 013410 (2000).

[26] T. P. de Silans, B. Farias, M. Oriá, and M. Chevrollier, Appl. Phys. B 82, 367 (2006).

[27] A. E. Afanasiev, P. N. Melentiev, and V. I. Balykin, J. Exp. Theor. Phys. Lett. 86, 172 (2007).

[28] A. E. Afanas'ev, P. N. Melent'ev, and V. I. Balykin, Bull. Russ. Acad. Sci. Phys. 72, 664 (2008).

[29] W. M. Soares, T. P. De Silans, M. Oriá, and M. Chevrollier, Int. J. Mod. Phys. A 24, 1764 (2009).

[30] K. P. Nayak, P. N. Melentiev, M. Morinaga, F. L. Kien, V. I. Balykin, and K. Hakuta, Opt. Express 15, 5431 (2007). 
[31] Z. W. Gortel, H. J. Kreuzer, and R. Teshima, Phys. Rev. B 22, 5655 (1980).

[32] H. J. Kreuzer and Z. Gortel, Physisorption Kinetics (Springer, Berlin, 1986).

[33] F. L. Kien, S. D. Gupta, and K. Hakuta, Phys. Rev. A 75, 062904 (2007).

[34] T. Nieddu, V. Gokhroo, and S. N. Chormaic, J. Opt. 18, 053001 (2016).

[35] P. Solano, J. A. Grover, J. E. Hoffman, S. Ravets, F. K. Fatemi, L. A. Orozco, and S. L. Rolston, in Advances in Atomic, Molecular, and Optical Physics, edited by E. Arimondo, C. C. Lin, and S. F. Yelin (Academic Press, New York, 2017), Vol. 66, pp. 439-505.

[36] K. P. Nayak, M. Sadgrove, R. Yalla, F. L. Kien, and K. Hakuta, J. Opt. 20, 073001 (2018).

[37] M. Stephens, R. Rhodes, and C. Wieman, J. Appl. Phys. 76, 3479 (1994).

[38] M. A. Bouchiat, J. Guéna, P. Jacquier, M. Lintz, and A. V. Papoyan, Appl. Phys. B 68, 1109 (1999).

[39] H. N. de Freitas, M. Oria, and M. Chevrollier, Appl. Phys. B 75, 703 (2002).

[40] D. Hümmer, P. Schneeweiss, A. Rauschenbeutel, and O. Romero-Isart, Phys. Rev. X 9, 041034 (2019).

[41] R. Pennetta, S. Xie, and P. S. J. Russell, Phys. Rev. Lett. 117, 273901 (2016).

[42] P. Solano, P. Barberis-Blostein, F. K. Fatemi, L. A. Orozco, and S. L. Rolston, Nat. Commun. 8, 1857 (2017).

[43] F. Le Kien and A. Rauschenbeutel, Phys. Rev. A 95, 023838 (2017).

[44] B. Olmos, G. Buonaiuto, P. Schneeweiss, and I. Lesanovsky, Phys. Rev. A 102, 043711 (2020).

[45] J. P. Dowling and J. Gea-Banacloche, Adv. At. Mol. Opt. Phys. 37, 1 (1996).

[46] F. Le Kien, V. I. Balykin, and K. Hakuta, Phys. Rev. A 70, 063403 (2004).

[47] S. Y. Buhmann, Dispersion Forces I: Macroscopic Quantum Electrodynamics and Ground-State Casimir, CasimirPolder and van der Waals Forces (Springer, Berlin, 2012).

[48] S. Fuchs, R. Bennett, R. V. Krems, and S. Y. Buhmann, Phys. Rev. Lett. 121, 083603 (2018).

[49] F. Le Kien, P. Schneeweiss, and A. Rauschenbeutel, Eur. Phys. J. D 67, 92 (2013).

[50] F. Le Kien, P. Schneeweiss, and A. Rauschenbeutel, Phys. Rev. A 88, 033840 (2013).

[51] E. Zaremba and W. Kohn, Phys. Rev. B 15, 1769 (1977).

[52] A. Zangwill, Physics at Surfaces (Cambridge University Press, Cambridge, England, 1988).

[53] H. Hoinkes, Rev. Mod. Phys. 52, 933 (1980).

[54] Precise calculations of the dispersion force need to account for the full complexity and imperfections of the atom-surface system [55]. While it is possible to calculate the exact form of the dispersion force between an atom and a dielectric cylinder from first principles [56-58], we are here mainly interested in scenarios where the dispersion force is only dominant at atomsurface separations smaller than the radius of the nanofiber. In this limit, the exact solution can be approximated by the nonretarded dispersion force near a half-space [46,57].

[55] G. L. Klimchitskaya, U. Mohideen, and V. M. Mostepanenko, Rev. Mod. Phys. 81, 1827 (2009).

[56] M. Schmeits and A. A. Lucas, Surf. Sci. 64, 176 (1977).
[57] M. Boustimi, J. Baudon, P. Candori, and J. Robert, Phys. Rev. B 65, 155402 (2002).

[58] V. M. Nabutovskii, V. R. Belosludov, and A. M. Korotkikh, J. Exp. Theor. Phys. 50, 352 (1979).

[59] A. D. McLachlan, Mol. Phys. 7, 381 (1964).

[60] J. M. Wylie and J. E. Sipe, Phys. Rev. A 30, 1185 (1984).

[61] N. P. Stern, D. J. Alton, and H. J. Kimble, New J. Phys. 13, 085004 (2011).

[62] Our findings do not change appreciably when using an exponential barrier $D \exp [-(r-R) p]$ instead of the polynomial in Eq. (2), for instance with $C / h=1.56 \mathrm{THz} \mathrm{nm}^{3}$, $V_{\min } / h=-128 \mathrm{THz}$, repulsive amplitude $D / h=1.6 \times$ $10^{6} \mathrm{THz}$, and decay length $p=53 \mathrm{~nm}^{-1}$ as suggested in Ref. [33].

[63] See Supplemental Material at http://link.aps.org/ supplemental/10.1103/PhysRevLett.126.163601 for an extended discussion of the photonic and phononic nanofiber modes, the calculation of the motional linewidths, and the heterodyne fluorescence spectroscopy scheme, which includes Refs. [64-78].

[64] J. D. Achenbach, Wave Propagation in Elastic Solids (North-Holland Publishing, Amsterdam, 1973).

[65] M. E. Gurtin, in Linear Theories of Elasticity and Thermoelasticity, Linear and Nonlinear Theories of Rods, Plates, and Shells, Mechanics of Solids, Vol. 2, edited by C. Truesdell (Springer, Berlin, 1984).

[66] C. Cohen-Tannoudji, J. Dupont-Roc, and G. Grynberg, Photons and Atoms: Introduction to Quantum Electrodynamics (Wiley-VCH, Weinheim, 2004).

[67] A. E. Armenàkas, D. C. Gazis, and G. Herrmann, Free Vibrations of Circular Cylindrical Shells (Pergamon Press, Oxford, 1969).

[68] R. J. Glauber and M. Lewenstein, Phys. Rev. A 43, 467 (1991).

[69] A. Messiah, Quantum Mechanics (Dover Publications, New York, 2014).

[70] J. I. Cirac, R. Blatt, P. Zoller, and W. D. Phillips, Phys. Rev. A 46, 2668 (1992).

[71] H.-P. Breuer and F. Petruccione, The Theory of Open Quantum Systems (Oxford University Press, Oxford, 2002).

[72] D. Reitz, C. Sayrin, R. Mitsch, P. Schneeweiss, and A. Rauschenbeutel, Phys. Rev. Lett. 110, 243603 (2013).

[73] B. Albrecht, Y. Meng, C. Clausen, A. Dareau, P. Schneeweiss, and A. Rauschenbeutel, Phys. Rev. A 94, 061401(R) (2016).

[74] J. I. Cirac, R. Blatt, A. S. Parkins, and P. Zoller, Phys. Rev. A 48, 2169 (1993).

[75] G. Sagué, E. Vetsch, W. Alt, D. Meschede, and A. Rauschenbeutel, Phys. Rev. Lett. 99, 163602 (2007).

[76] B. D. Patterson, P. Solano, P. S. Julienne, L. A. Orozco, and S. L. Rolston, Phys. Rev. A 97, 032509 (2018).

[77] M. Lindberg, Phys. Rev. A 34, 3178 (1986).

[78] C. Cohen-Tannoudji, J. Dupont-Roc, and G. Grynberg, Atom-Photon Interactions: Basic Processes and Applications (Wiley-VCH, Weinheim, 1998).

[79] Handbook of Optics: Devices, Measurements, and Properties, 2nd ed., edited by M. Bass, E. W. Van Stryland, D. R. Williams, and W. L. Wolfe (McGraw-Hill, New York, 2001), Vol. 2.

[80] This is the largest radius compatible with the single-mode regime for the light fields of the two-color trap. 
[81] We use the commercial COMSOL Multiphysics ${ }^{\circledR}$ software package [82]. Unbound states are obtained by approximating free space with an interval sufficiently large so as not to influence any of the results presented in this Letter.

[82] COMSOL Inc., COMSOL Multiphysics Reference Manual, Version 5.2a (COMSOL Inc., Stockholm, 2016).

[83] J. Meija, T. B. Coplen, M. Berglund, W. A. Brand, P. D. Bièvre, M. Gröning, N. E. Holden, J. Irrgeher, R. D. Loss, T. Walczyk, and T. Prohaska, Pure Appl. Chem. 88, 265 (2016).

[84] T. R. Meeker and A. H. Meitzler, in Methods and Devices, Part A, Physical Acoustics: Principles and Methods, edited by W. P. Mason (Academic Press, New York, 1964), Vol. I A, pp. 111-167.
[85] P. S. Jessen, C. Gerz, P. D. Lett, W. D. Phillips, S. L. Rolston, R. J. C. Spreeuw, and C. I. Westbrook, Phys. Rev. Lett. 69, 49 (1992).

[86] D. Marcuse, Light Transmission Optics (Van Nostrand Reinhold, New York, 1982).

[87] A. W. Snyder and J. Love, Optical Waveguide Theory (Springer, New York, 2012).

[88] F. Le Kien, J. Q. Liang, K. Hakuta, and V. I. Balykin, Opt. Commun. 242, 445 (2004).

[89] C. Wuttke and A. Rauschenbeutel, Phys. Rev. Lett. 111, 024301 (2013).

[90] Y. Meng, A. Dareau, P. Schneeweiss, and A. Rauschenbeutel, Phys. Rev. X 8, 031054 (2018). 